\section{The Impact of Routine Cardiology Consultation on Critically III Patients with Elevated Troponin Levels}

\begin{abstract}
Authors: *Omar Kousa, ${ }^{1}$ Amr Essa, ${ }^{1}$ Mohammed Saleh, ${ }^{1}$ Muhammad Ahsan, ${ }^{1}$ Yaman Alali, ${ }^{1}$ Venkata Pajjuru, ${ }^{1}$ Abedelrahman Anani, ${ }^{1}$ Aiza Ahmad, ${ }^{1}$ Janani Baskaran, ${ }^{1}$ Ryan W. Walters, ${ }^{2}$ Arindam Sharma, ${ }^{3}$ Toufik M. Haddad, ${ }^{3}$ Aiman Smer ${ }^{3}$

1. Creighton University, Internal Medicine, Omaha, Nebraska, USA

2. Creighton University, Omaha, Nebraska, USA

3. Creighton University, Cardiology Department, Omaha, Nebraska, USA

*Correspondence to mr.koosaa2009@hotmail.com
\end{abstract}

Disclosure: The authors have declared no conflicts of interest.

Keywords: Cardiology, consult, critical care, troponin.

Citation: EMJ Cardiol. 2020;DOI/10.33590/ emjcardiol/2009141

\section{BACKGROUND AND AIMS}

Cardiac troponin (cTn) is used to diagnose acute coronary syndrome (ACS). However, cTn can also be elevated in critically ill patients secondary to demand ischaemia or myocardial injury. In a meta-analysis of 20 different studies involving critically ill patients, cTn was found to be elevated in $43 \%$ of patients. ' Prior studies have evaluated the significance of cTn as a prognostic factor and mortality predictor in different clinical entities in intensive care units (ICU)., ${ }^{2,3}$ However, the data on the impact of cardiology consultation on the outcomes in this specific context of critical care patients are limited. While prior studies have addressed such an impact on the outcomes among different groups of surgical patients, ${ }^{4,5}$ to the authors' knowledge, no prior studies have examined that impact on critically ill patients with elevated cTn. This study aimed to investigate the impact of cardiology consultation on critically ill patients in the medical ICU with elevated cTn and no signs of ACS.

\section{MATERIALS AND METHODS}

A retrospective analysis was conducted of all patients admitted to the medical ICU with elevated cTn levels without ACS diagnosis, between January 2013 and December 2018. Patients who were admitted with ACS as a primary diagnosis, had ECG findings of ischaemic changes, had new ST-elevation myocardial infarction, or had new left bundle branch block were excluded. Patients were divided into two groups based on documentation of cardiology consultation. The primary endpoint was to assess in-hospital, 30-day, and 1-year mortality. Secondary outcomes were the length of stay, further cardiac testing, and 30-day readmission rate and new prescription of cardiac medications. Multivariable logistic regression models were estimated for mortality, readmission, and additional cardiac testing, whereas KaplanMeier and multivariable Cox proportional hazard models were estimated for the probability of discharge to allow censoring as a result of death during the index hospitalisation.

\section{RESULTS}

A total of 766 patients met inclusion criteria, of whom 484 (63.2\%) received cardiology consultation. Age and history of coronary artery disease were independently associated with greater odds of cardiology consult with adjusted odds ratios (aOR) of 1.02 (95\% confidence interval [Cl]: 1.00-1.04) and 2.2 (95\% $\mathrm{Cl}$ : 1.3-3.8), respectively. Patients with cardiology consultation had similar unadjusted in-hospital and 30-day mortality rates $(p=0.667$ and $p=0.959$, respectively), but had higher unadjusted 1-year mortality $(p=0.041)$. However, after adjusting for patient demographic and clinical characteristics, no differences in any mortality outcomes were observed (Figure 1). Patients with cardiology consultation had a higher adjusted odds ratio of all-cause 30-day readmission (aOR: 3.5; 95\% Cl: 1.6-7.6; $p=0.001$ ), additional cardiac testing (aOR: 3.34; 95\% Cl: 1.9-6.0; p<0.001), new cardiac medications (aOR: 5.9; 95\% Cl: 3.5-9.9; $\mathrm{p}<0.001$ ), but not cardiac-specific 30-day readmission (aOR: 3.2; 95\% Cl: 0.7-14.9; $\mathrm{p}=0.137$ ). 


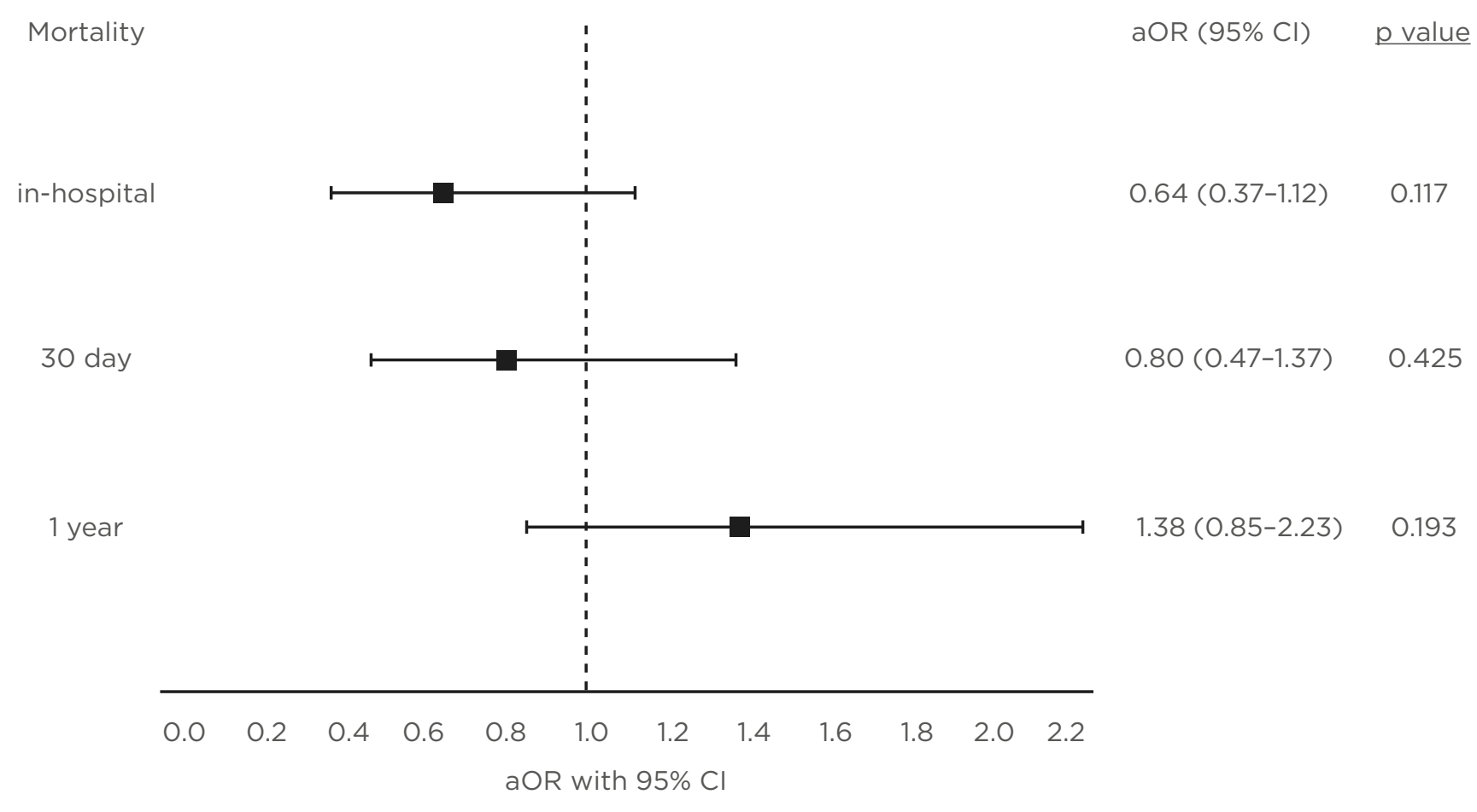

\section{Figure 1: Mortality outcomes.}

Forest plot showing adjusted odds ratios for mortality outcomes. All models adjusted for age, baseline ejection fraction, ischaemia, peak troponin, hypertension, diabetes, chronic kidney disease, hyperlipidaemia, smoking status, alcohol abuse, substance abuse, as well as a history of coronary artery disease, atrial fibrillation, valve disorder, or heart failure. Odds ratios $<1$ favour patients who received the cardiology consultation. Error bars represent 95\% confidence intervals.

aOR: adjusted odds ratio; $\mathrm{Cl}$ : confidence interval.

Finally, although length of stay was statistically longer in patients with cardiology consultation, both unadjusted and adjusted probability of discharge during the index hospitalisation was not statistically different (unadjusted $p=0.188$; adjusted hazard ratio: 0.8; 95\% Cl: 0.7-1.1; $\mathrm{p}=0.129$ ).

\section{CONCLUSION}

This study suggests that cardiology consultation was not associated with improved outcomes and led to a longer length of stay resulting in higher cost of hospitalisation. Cardiology consultation should be ordered judiciously and in the right clinical context in the ICU to avoid excessive diagnostic testing and interventions which may not be beneficial to the patient.

\section{References}

1. Lim W et al. Elevated cardiac troponin measurements in critically ill patients. Arch Inter Med. 2006;166(22):244654.

2. Becattini $C$ et al. Prognostic value of troponins in acute pulmonary embolism. Circulation. 2007;116(4):427-33.

3. Lazzeri $\mathrm{C}$ et al. Serial measurements of troponin and echocardiography in patients with moderate-to-severe acute respiratory distress syndrome. J Crit Care. 2016;33:132-6.

4. Troisi $\mathrm{N}$ et al. Preoperative cardiac assessment in patients undergoing major vascular surgery. Vascular. 2011;19(4):178-86.

5. Squizzato $F$ et al. Clinical impact of routine cardiology consultation prior to elective carotid endarterectomy in neurologically asymptomatic patients. Eur J Vasc Endovasc Surg. 2020;59(4):536-44. 